\title{
CONDUTAS DE URGÊNCIA NAS SÍNDROMES ISQUÊMICAS MIOCÁRDICAS INSTÁVEIS
}

\author{
ACUTE CORONARY SYNDROMES IN THE EMERGENCY DEPARTMENT
}

José Antonio Marin-Neto ${ }^{1}$; Benedito Carlos Maciel' ${ }^{1}$ Antonio Pazin Filho ${ }^{1} \&$ Renato Barroso P. Castro²

\begin{abstract}
'Docentes. Divisão de Cardiologia. Departamento de Clínica Médica. Faculdade de Medicina de Ribeirão Preto - USP. ${ }^{2}$ Médico Assistente. Divisão de Cardiologia. Hospital das Clínicas da Faculdade de Medicina de Ribeirão Preto - USP.

CorRespondência: Prof. Dr. José Antonio Marin Neto. Divisão de Cardiologia. Departamento de Clínica Médica. Faculdade de Medicina de Ribeirão Preto - USP - CEP: 14048-900 - Ribeirão Preto - SP
\end{abstract}

MARIN-NETO JA; MACIEL BC; PAZIN FILHO A \& CASTRO RBP. Condutas de urgência nas síndromes isquêmicas miocárdicas instáveis. Medicina, Ribeirão Preto, 36: 187-199, abr./dez. 2003.

RESUMO - As síndromes isquêmicas miocárdicas Instáveis (SIMI) são freqüentes na Sala de Urgência (SU) e o seu não reconhecimento ou tratamento inadequado implicam em elevada morbimortalidade. A conduta, no ambiente da SU, é dependente do risco, estimado a partir de aspectos de história e exame físico, parâmetros eletrocardiográficos e da dosagem de marcadores séricos de necrose miocárdica. Os princípios terapêuticos das SIMIs com supradesnivelamento de ST ao eletrocardiograma (infarto agudo do miocárdio) e das SIMIs sem supradesnivelamento de ST (angina instável e infarto agudo sem supradesnível de ST) são revisados neste artigo.

UNITERMOS - Isquemia Miocárdica. Infarto do Miocárdio. Angina Instável.

\section{1- INTRODUÇÃO}

As síndromes isquêmicas miocárdicas instáveis (SIMIs) manifestam-se, caracteristicamente, por aguda dor torácica, de duração variável, nas últimas 24 h, e constituem dos mais comuns e graves problemas clínicos em unidades de emergências (U.E.s) médicas. Assim, até 7\% de todos os casos, atendidos em U.E., relacionam-se a queixas de dor torácica. Do total de casos atendidos com dor torácica, nas UEs ( 5 a 8 milhões/ano, nos EUA), 70 a $75 \%$ não apresentam isquemia miocárdica, mas, do restante, 10 a $15 \%$ têm infarto e 10 a 15\%, angina instável, as duas vertentes fundamentais das SIMIs. Representam problema de complexa abordagem, com taxas de internação hospitalar da ordem de 50 a $60 \%$; ainda para depor sobre a complexidade desse contexto, assinala-se que entre 2 e $8 \%$ dos pacientes com infarto do miocárdio são liberados da UE ou Centro de dor torácica (CDT) para casa, sem o diagnóstico correto. Portanto, requer-se abordagem institucional, que contemple a imperiosidade de: - promover uma avaliação sistematizada de pacientes com dor torácica aguda;

- aprimorar a acuracidade e agilizar o diagnóstico das SIMIs;

- reduzir o tempo para tratamento e custos dessa investigação diagnóstica.

\section{2- ESTRATÉGIA BÁSICA, DE ABORDAGEM CLÍNICA}

Em face da maior prevalência e dos elevados níveis de mortalidade e morbidade, a estratégia inicial de atendimento de pacientes com dor torácica aguda deve considerar a síndrome isquêmica aguda SIMI como a primeira hipótese a ser confirmada ou afastada, mesmo na presença de sintomatologia não muito típica. 
Objetivos da estratégia de atendimento

1. Distinguir os pacientes com SIMI daqueles com outras condições potencialmente graves (dissecção da aorta, embolia pulmonar, pericardite) ou causas menos graves de dor torácica (Tabela I);

2. avaliar o risco potencial de desfechos adversos (Tabela II);

3. iniciar, rapidamente, o tratamento em pacientes com risco elevado.

Entre as medidas iniciais de atendimento, incluem-se as relacionadas a seguir.

- história clínica dirigida e exame físico;

- obter acesso venoso periférico;

- administração de aspirina AAS (200mg, mastigáveis, para absorção pela mucosa bucal). Inibidor da ativação plaquetária interfere no processo agudo de trombose coronariana, demonstrando benefícios consistentes sobre mortalidade (imediata e tardia) e morbidade (taxa de infarto e reinfarto);

- administração de oxigênio (quatro L/min, cateter nasal);

- Realizar eletrocardiograma (ECG) de 12 derivações.

- Um teste com dinitrato de isossorbitol $(5 \mathrm{mg} / \mathrm{sublin}$ gual) pode ser realizado, desde que PAS $>90 \mathrm{mmHg}$, não haja suspeita de infarto de VD, e, preferencialmente, após o ECG seja revelado que não existe supradesnivelamento de ST em parede inferior do VD (Vide abaixo). É um teste extremamente valioso para pacientes com suspeita de síndrome de Prinzmetal, que podem, inclusive, apresentar-se com supradesnivelamento de ST: o nitrato reverte o espasmo, e essa alteração de ST, poupando o paciente de receber tratamento trombolítico;

- coleta de sangue para dosagem de marcadores de lesão miocárdica (idealmente, um marcador de elevação rápida - mioglobina - associado a um marcador de elevação e queda mais lenta - CK-MB massa ou troponina).

\section{Essas medidas devem ser executadas em até 10 min desde a entrada do paciente na unidade de emergência.}

Dentro de 30 minutos, desde a entrada do paciente, com base nos aspectos clínicos do paciente, incluindo a análise do ECG e, se possível, a dosagem de entrada dos marcadores bioquímicos de lesão miocárdica:

- reavaliar a probabilidade de SIMI e o risco de óbito ou IAM não fatal -Tabela II;

- analisar o ECG e classificá-lo de acordo com a presença de novo bloqueio de ramo esquerdo, presen-

Tabela I - Estimativa da probabilidade de SIMI com base em manifestações clínicas

\section{Alta probabilidade}

Presença de qualquer das seguintes características

\section{Probabilidade intermediária Ausência das anteriores e presença de qualquer das seguintes características}

- Dor ou desconforto precordial ou no braço esquerdo, reproduzindo angina previamente documentada

- História de coronariopatia conhecida, incluindo IAM

- Insuficiência mitral transitória, hipotensão, diaforese, edema pulmonar ou estertores pulmonares

- Dor precordial + alterações novas, ou presumivelmente novas, transitórias, do segmento ST (desvios $\geq 0,5$ $\mathrm{mm}$ ) ou da onda $\mathrm{T}$ (inversão $\geq 2 \mathrm{~mm}$ )

- Níveis elevados de troponinas ou CK-MB
- Dor ou desconforto precordial ou no braço esquerdo, como principal sintoma

- Idade > 70 anos

- Gênero masculino

- Diabetes mellitus

- Doença vascular periférica

- Presença de ondas Q no ECG

- Presença de anormalidades de ST ou

T no ECG, não documentadas como recentes
Baixa Probabilidade Ausência das anteriores e presença de qualquer das seguintes caracterís ticas

- Dor precordial atípica

- Uso recente de cocaína

- Desconforto torácico, reproduzido pela palpação

- Achatamento ou inversão de onda T em derivações com ondas $\mathrm{R}$ dominantes

- Dor precordial + ECG normal 
Tabela II - Estimativa de risco de óbito ou IAM não fatal no curto prazo, em pacientes com SIMI

Alto risco

Presença de qualquer das seguintes características
Risco intermediário Ausência das anteriores e presença de qualquer das seguintes características

- Previamente: IAM, doença cerebrovascular ou periférica, cirurgia de revascularização miocárdica ou uso de AAS

- Dor precordial de repouso, ocorrendo há mais de $20 \mathrm{~min}$

- Edema pulmonar, provavelmente devido a isquemia

- Aparecimento ou piora de sopro de insuficiência mitral

- Presença de $\mathrm{B}_{3}$ ou aparecimento ou piora de estertores pulmonares

- Hipotensão arterial, bradicardia, taqui-cardia

- Idade > 75 anos

- Dor precordial + alterações transitórias do segmento $\mathrm{ST} \geq 0,5 \mathrm{~mm}$

- Bloqueio de ramo esquerdo, novo ou presumivelmente novo

- Taquicardia ventricular sustentada

- Níveis muito elevados de troponinas
- Idade > 70 anos

- Inversão de onda T > 2mm no ECG

- Presença de ondas Q patológicas no ECG

- Aumento discreto de troponinas
Baixo Risco

Ausência das anteriores e presença de qualquer das seguintes características

- Angina de início recente, Classe III ou IV, nas duas últimas semanas, sem dor de repouso prolongada (>20min), mas com probabilidade alta ou moderada de coronariopatia

- ECG normal ou inalterado durante episódio de desconforto precordial

- Marcadores bioquímicos, de lesão miocárdica, normais. ça de elevação de segmento S-T, de depressão de segmento S-T ou inversão de onda T, ou, ainda, de ausência de alterações sugestivas de isquemia miocárdica;

- repetir ECG, em 15 ou 30 min, quando exame inicial não mostrou sinais de isquemia, mas há probabilidade elevada de isquemia miocárdia ou dor persistente;

- na dependência da presença e da intensidade da dor torácica, considerar a analgesia com meperidina (20-50 mg/endovenosa (EV); se necessário, repetir em até 30 min, e, após, a cada 3-4 h; via EV: infusão lenta; menor dose em idosos) ou morfina (1-5 $\mathrm{mg} / \mathrm{EV}$, se necessário, repetir em até $30 \mathrm{~min}$ e, após, a cada 4 h; via EV: infusão lenta; menor dose em idosos, atenção para insuficiência respiratória);

- iniciar tratamento específico, incluindo técnicas de reperfusão aguda, quando indicadas, para os pacientes com síndromes características (Vide adiante);
- transferir pacientes de alto risco para unidade de observação, sob monitorização eletrocardiográfica, contínua.

As dosagens de marcadores enzimáticos deverão ser repetidas com 8,12 e $18 \mathrm{~h}$.

Pacientes com suspeita clínica de infarto agudo do miocárdio, com elevação de segmento S-T ou de angina instável/infarto sem elevação de S-T, deverão seguir as recomendações expressas a seguir.

Pacientes com baixa probabilidade e/ou baixo risco, sem alterações eletrocardiográficas, sugestivas de isquemia, ou com alterações inespecíficas, que não preencham os critérios de angina instável, com padrão de início recente, deverão:

- manter-se em observação por 8 a 12 h;

- ter outros ECGs de repouso, com 4, 8 e 12 h ou mais precocemente, na dependência da persistência de dor precordial; 
- ter dosagens de marcadores de lesão miocárdica com 8 e 12 h;

- submeter-se a ecocardiografia bidimensional, para identificação de anormalidades de mobilidade segmentar do VE, ou cintilografia miocárdica de perfusão sob estresse vasodilatador, para comprovar ou afastar a presença de áreas miocárdicas com redução da reserva vasodilatadora;

- dependendo da probabilidade clínica de doença isquêmica, realizar teste de estresse precocemente ou encaminhar para seguimento ambulatorial.

Considerar as possíveis causas de dor torácica de origem não cardíaca, e encaminhar o paciente para avaliação específica.

\section{3- CONDUTAS NAS SIMIS SEM SUPRA- DESNIVELAMENTO MANTIDO DE ST}

Esta categoria abrange os quadros de Angina Instável (AI) e infarto agudo do miocárdio sem supradesnivel de ST (IAM $\underline{S} S S T$ )

- Definição: dor precordial, sugestiva de isquemia, com pelo menos uma das seguintes características: 1) ocorrência em repouso (ou com mínimo esforço), com duração maior que $>20$ min, 2) dor intensa e descrita como de início recente ( $<1$ mês) ou 3) caráter progressivo (em intensidade, duração e frequiência de episódios).

- Entre as causas de atendimento por dor torácica aguda, essas síndromes constituem fração significante, e, atualmente, majoritária (>60\%), relativamente ao conjunto das SIMIs.

Sua fisiopatologia baseia-se, na maioria das vezes, na em formação de trombo não oclusivo sobre placa arterial aterosclerótica, acarretando significativa limitação de fluxo coronário subepicárdico, mesmo em repouso. O espasmo vascular, na região acometida, ocorre em graus variáveis; quando representa mecanismo primordial (espasmo sobre placa discreta ou mesmo não saliente na luz vascular), configura-se a chamada angina vasoespástica ou de Prinzmetal.

- Como já ressaltado, no atendimento das SIMIs a prioridade inicial é confirmar/afastar a presença de infarto agudo do miocárdio com supra-desnível de S-T, o que implica em iniciar, ou não, imediatamente, terapêutica de reperfusão (Vide abaixo). Uma vez afastada essa possibilidade, a diferenciação ulterior entre o IAM $\underline{S} S S T$ e a AI será fundamentada na elevação (infarto) ou não (AI) dos marcadores séricos de lesão miocárdica (troponinas, CK-MB) através de avaliação laboratorial, seriada. Entretanto, para fins práticos de conduta terapêutica, sua abordagem inicial se faz de modo semelhante.

História de eventos coronarianos prévios (IAM, angina estável ou instável) é comum no paciente que se apresenta com AI/IAMSSST; a ocorrência, nos primeiros seis meses após intervenção coronariana, percutânea, sugere reestenose da lesão tratada enquanto que, em período > seis meses, a ocorrência de nova obstrução é mais provável. A presença de fatores de risco deve ser pesquisada (para avaliar a probabilidade de DAC), e pode, também, relacionar-se a maior risco de evolução para IAM ou óbito (critérios TIMI).

O exame físico pode ser normal ou revelar alterações sugestivas de grande área miocárdica em risco, tais como: palidez cutânea, surgimento de B3 ou B4, taquicardia, regurgitação mitral, sinais de congestão pulmonar ou mesmo de baixo débito.

O ECG é recurso diagnóstico imprescindível na avaliação e seguimento dos pacientes; deverá ser realizado dentro dos primeiros $10 \mathrm{~min}$ da chegada do paciente ao serviço médico de urgência. É importante saber que o primeiro exame poderá ser normal em até $50 \%$ dos casos, o que salienta a importância da realização de exames seriados. A comparação com traçados prévios de que o paciente disponha é de extrema utilidade na detecção de novas alterações isquêmicas. Na AI, a ocorrência de depressão de segmento S-T (ou mesmo supradesnível transitório) e inversão de onda T observa-se em até $50 \%$ dos casos; detecção de alterações dinâmicas de S-T tem implicações prognósticas, enquanto que as de onda $\mathrm{T}$ são mais sensíveis, porém, menos específicas. Quando pronunciadas, entretanto (inversões $\geq 3 \mathrm{~mm}$ ), acarretam pior prognóstico. A monitorização contínua do ECG (e do segmento S-T), nas primeiras $24 \mathrm{~h}$, parece ser mais sensível que as alterações sintomáticas para afastar/ confirmar isquemia miocárdica e encerra valor prognóstico, além de detectar bloqueios de ramo, distúrbios de condução $\mathrm{A}-\mathrm{V}$, e arritmias diversas.

Tradicionalmente, a utilização da creatinoquinase MB (CK-MB) tem embasado o diagnóstico da lesão isquêmica do miocárdio; a preferência atual é para a medida de sua concentração plasmática total (CK-MB massa), em lugar da medida de sua atividade, com sensibilidade de $97 \%$ e sensibilidade de $90 \%$ 
para o diagnóstico do IAM. Pode estar aumentada em situações de lesão da musculatura lisa ou esquelética. É útil no diagnóstico de IAMS $S S T$ e deverá ser realizada na chegada e a cada $8 \mathrm{~h}$ (pelo menos duas amostras, se não há elevação, ou até a normalização dos valores).

As troponinas são proteínas da estrutura contrátil do miócito e, portanto, ausentes da circulação em condições normais. O desenvolvimento de anticorpos monoclonais para detecção da Troponina I (TnI) e $\mathrm{T}(\mathrm{Tn} T)$ acrescentou ao arsenal diagnóstico um exame altamente sensível para detecção de lesão miocárdica; sua elevação, em nível discreto $(<0.1)$ sem elevação de CK-MB, caracteriza, atualmente, o que se convencionou chamar de microinfartos. Essa distinção parece se justificar pela ainda discutível, mas provável, associação desse quadro a prognóstico mais reservado do que a SIMI sem essa elevação mínima de tropononinas, aproximando-o da evolução do IAMSSSST. Embora não se elevem mais precocemente que a CK-MB, as troponinas permanecem em circulação por mais tempo, permitindo o diagnóstico retrospectivo de síndromes isquêmicas com maior tempo de retardo (até sete dias para TnI e até 14 dias para TnT). Em contraposição, as troponinas perdem para a CK-MB, quando se trata de confirmar a ocorrência de reinfarto.

A mioglobina é um marcador de necrose do miócito, não específico para a fibra miocárdica, que apresenta, como única vantagem, a elevação precoce após o evento isquêmico (início de elevação em torno de $3 \mathrm{~h}$ e pico em $12 \mathrm{~h}$ ); sua maior utilidade está na exclusão do diagnóstico de IAM nos em pacientes que se apresentam precocemente à UE; não deve ser utilizada como único marcador sérico.

Imageamento cardíaco: Ecocardiograma e Cintilografia Miocárdica de Perfusão (CMP): são recursos diagnósticos, de utilização crescente nas UEs e CDTs; são úteis, notadamente, nos pacientes com ECG não diagnóstico ou na presença de BRE ou de ritmo de marca-passo artificial. Ambos acrescentam dados sobre a função contrátil do ventrículo esquerdo, dadoaspecto de utilidade prognóstica e na condução terapêutica. A CMP em repouso, realizada na vigência da dor torácica ou logo após sua ocorrência pode confirmar ou afastar a etiologia isquêmica precocemente. A detecção de anomalias de contratilidade segmentar em repouso, pelo ECO, pode ser utilizada com a mesma finalidade, desde que respeitada a proximidade temporal em relação à dor.
Testes de esforço com monitorização de ECG são úteis e seguros em indivíduos admitidos em CDT ou UE para esclarecimento de dor torácica, estratificados como de baixo risco (ECG e marcadores séricos inalterados). Realizados após 12 a 24 h de estabilização clínica, é útil na confirmação/ exclusão precoce do diagnóstico, evitando, muitas vezes, internações prolongadas e desnecessárias. Recomenda-se a utilização de protocolos atenuados ou de rampa, respeitando-se rigorosamente todos os procedimentos de segurança.

\section{Encaminhamento genérico, mas específico, para pacientes com SIMISSST:}

A definição das condutas a serem tomadas dependerá, nesse ponto, da correta estratificação do paciente: com base nos aspectos dda anamnese, exame físico e exames complementares, estima-se a probabilidade de que o quadro seja realmente de SIMISSST e risco imediato de eventos graves (morte, infarto agudo do miocárdio),

Os pacientes considerados como de baixo risco (mas com probabilidade de SIMI moderada ou elevada) deverão ser mantidos em observação na UE/CDT pelo período mínimo de $8 \mathrm{~h}$; nesse prazo, serão submetidos a dosagens de marcadores de lesão miocárdica e eletrocardiogramas seriados. Caso não surjam evidências de isquemia, o paciente poderá ser submetido à teste de estresse físico em esteira ergométrica, já na própria internação, ou a critério clínico, encaminhado para avaliação ambulatorial.

Todos os casos considerados como de risco alto ou intermediário deverão, preferencialmente, ser transferido para ambiente de terapia intensiva ou unidade coronariana (UCo) onde permanecerão até estabilização completa. Caso a opção seja por estudo angiográfico precoce, para provável angioplastia o paciente retornará à UCo após o procedimento; se a decisão fôr por tratamento clínico, permanecerá na UCo de 24 a 48 h após estabilização, sendo transferido para a enfermaria, de preferência, já sem medicações endovenosas. Se, após a cinecoronariografia, a opção fôr por revascularização cirúrgica precoce, o paciente aguardará o procedimento, também, na UCo. Obviamente, ao classificar-se um paciente como de alto risco, a abordagem diagnóstica e terapêutica priorizará procedimentos de alta confiabilidade e que possam, rapidamente, definir a gravidade e indicar a melhor opção para o caso. A angiografia, ao definir a anatomia coronariana, avaliar a função ventricular, identifi- 
car lesões, requerendo procedimentos urgentes (lesões de tronco de coronária esquerda, sub-oclusivas proximais, em ramos calibrosos e extensos, etc) impõe-se, nesse contexto, como o exame de escolha. Embora não necessariamente apresentando-se como risco elevado, os pacientes com cirurgia de revascularização prévia tendem a ser estudados por esse método. Uma vez indicado o estudo intravascular, outra decisão de igual importância refere-se ao momento da realização do referido exame: em pacientes já estabilizados, em geral, opta-se pela realização dele após as primeiras $48 \mathrm{~h}$, o que permite que o paciente seja levado à mesa de cateterismo mais estável clinicamente. Obviamente, se a estabilização mostrar-se difícil, com persistência da dor ou instabilidade hemodinâmica, o exame intravascular passa a ser indicado de imediato.

\section{Condutas terapêuticas específicas}

a) Oxigenioterapia - como delineado acima, em condutas gerais.

\section{b) Analgesia}

A persistência da dor anginosa, após o início do tratamento medicamentoso, é totalmente indesejável, visto aumentar o consumo de oxigênio, a incidência de arritmias, etc. Nos pacientes com SIMI- SSST, proceder como delineado acima, mas observando-se que, diversamente do que ocorre em pacientes com IAM-CSST, que quase sempre requerem analgesia intensa, os nitratos e betabloqueadores são efetivos, quase sempre, para aliviar o sintoma. O uso de ansiolíticos, principalmente os benzodiazepínicos, é freqüente, mas estimula-se a individualização dessa conduta.

\section{c) Nitratos}

O uso dos nitratos baseia-se mais no empírico reconhecimento de sua capacidade de alívio da dor isquêmica do que em estudos randomizados, com evidências sobre benefício prognóstico. Sua ação antiisquêmica é explicada pela redução do retorno venoso além de vasodilatação coronariana direta. A administração deverá ser iniciada precocemente por via sublingual (nitroglicerina $0.4 \mathrm{mg}$, dinitrato de isosorbida, $5 \mathrm{mg}$, a cada $3 \mathrm{~h}$ ), e, quase sempre, mantida por via endovenosa (nitroglicerina ou mononitrato). Não iniciar nitratos, se pressão arterial for inferior a $100 \mathrm{mHg}$ ou se houver história de uso de sildenafil ou análogos nas últimas $24 \mathrm{~h}$ (risco de hipotensão grave). A administração EV deverá ser titulada até se obter o controle dos sintomas, ou queda $>20 \mathrm{mmHg}$ na PA sistólica. Usualmente, continua-se com administração por via oral cerca de $48 \mathrm{~h}$ após o controle da dor, mas com observância do preceito de evitar o uso contínuo, para evitar taquifilaxia.

\section{d) Bloqueadores betaadrenérgicos}

Embora com menor grau de evidência que no IAM, estão bastante bem comprovados os benefícios dos betabloqueadores na angina instável, através da redução de eventos maiores (óbitos e evolução para IAM) ou angina recorrente; sua ação anti-isquêmica baseia-se em redução da freqüência cardíaca, da pressão arterial sistêmica e da contratilidade miocárdica e conseqüente redução no consumo de $\mathrm{O} 2$ miocárdico, além de provável proteção contra arritmias.

São fármacos de primeira linha nas SIMIISSSTs e só não deverão ser utilizadas frente a contra-indicações evidentes: bloqueio atrioventricular, bradicardia significante, hipotensão persistente, edema pulmonar ou broncoespasmo grave. Na presença de dor ou isquemia evidente, deverão ser iniciados por via venosa e, posteriormente, continuados por a via oral. Não se comprovou superioridade de um fármaco sobre outro da mesma classe, devendo a escolha basear-se na farmacodinâmica, custo e familiaridade com o uso; as dosagens propostas para o metoprolol e atenolol, dotados de certa seletividade para os receptores beta-1, são:

Metoprolol: $\mathrm{EV}-5 \mathrm{mg}(1 \mathrm{~min}-2 \mathrm{~min})$ a cada $5 \mathrm{~min}$ até completar a dose máxima de $15 \mathrm{mg}$. VO - 50mg-100mg a cada $12 \mathrm{~h}$, iniciada 15min após a última administração EV.

Atenolol: $\mathrm{EV}-5 \mathrm{mg}(1 \mathrm{~min}-2 \mathrm{~min})$ a cada $5 \mathrm{~min}$ até completar a dose máxima de $10 \mathrm{mg}$. VO - 25mg-50mg a cada $12 \mathrm{~h}$, iniciada 15 min após a última administração EV.

e) Medicamentos antiplaquetários

1) AAS - por sua ação antitrombótica, bem conhecida e por efeitos bem documentados na redução da evolução para IAM e óbito, constitui primeira linha no tratamento da SIMISSST;

2) Tienopiridínicos - o clopidogrel e a ticlopidina estão disponíveis nesse grupo; por sua ação antiplaquetária, inibindo a ativação mediada por ADP, fundamenta-se a associação ao AAS, tendo sido comprovado nítido benefício em termos de redução de eventos graves, em pacientes com risco moderado/elevado. A 
ticlopidina tem efeito pleno somente após vários dias, e, também, provoca, comparativamente, mais problemas colaterais - neutropenia em $2.4 \%$ (grave em $0.8 \%$ ), dores abdominais, náuseas e vômitos e, raramente, púrpura trombocitopênica idiopática, o que limita sua utilização. Sua dosagem é de $250 \mathrm{mg}$, via oral, a cada $12 \mathrm{~h}$. Acompanhamento do leucograma deverá ser realizado nos primeiros três meses, a cada 30 dias. Com melhor perfil de segurança, o clopidogrel firmou-se como o mais indicado em associação ao AAS, inclusive para pacientes tratados agudamente com intervenção coronariana percutânea (ICP). Preconiza-se dose de ataque de $300 \mathrm{mg}$, via oral e manutenção de $75 \mathrm{mg} /$ dia; o período de prescrição ainda está indefinido, porém há demonstração de benefício até em torno de nove meses. Salienta-se a necessidade de suspensão do clopidogrel com antecedência mínima de cinco dias, caso o paciente receba indicação de revascularização cirúrgica, dado o risco de sangramento grave perioperatório. Em condições de emergência, deve-se recorrer à transfusão de plaquetas.

\section{f) Medicamentos antitrombínicos}

1) Heparinas: a serem empregadas em todos os pacientes de alto risco, e em muitos daqueles com risco moderado, durante a hospitalização, antagonizam os fatores IIa (trombina), IXa e Xa. Impedem a progressão do trombo já formado, porém não promovem a lise do mesmo. A heparina convencional, (não fracionada) constitui mistura de moléculas de tamanhos variados (5.000 a 30.000 dáltons), com ação anticoagulante variável e com alta taxa de ligação a proteínas plasmáticas e células endoteliais. As heparinas de baixo peso molecular são obtidas através da despolimerização das cadeias polissacarídicas de heparina, com redução do seu tamanho médio (ao redor de 6.000 daltons). Suas características principais são menor ligação a proteínas e células endoteliais, maior biodisponibilidade e conseqüente efeito anticoagulante mais previsível e sustentado, com possibilidade de administração duas vezes ao dia e sem a necessidade de monitorização laboratorial. Por não serem capazes de inativar o fator IIa, não alteram significantemente as provas de coagulação. As heparinas de baixo peso molecular sofrem maior influência da função renal, devendo ter suas dosagens reduzidas com clearance inferior a $30 \mathrm{ml} / \mathrm{min}$; salienta-se, ainda, sua menor reversibilidade com o uso da protamina, em relação a heparina convencional.

A heparina não fracionada é utilizada $\mathrm{EV}$, na dose de ataque de 50-60 U/Kg (Máximo de 5.000UI), seguida de 12 a 15 UI/Kg/h (máximo de 1.000 UI/h), monitorizando-se com o TTPA (tempo de tromboplastina parcial ativada), objetivando-se prolongação de 1.5 a 2 vezes o tempo basal (50 a 70s). Os estudos que analisaram as heparinas de baixo peso, disponíveis (nadroparina, dalterparina, fraxiparina e enoxaparina) revelaram resultados relativamente heterogêneos, com possível superioridade da enoxaparina sobre a heparina não fracionada, em termos de redução de morte, IAM não fatal e recorrência de isquemia. Há, também, análises de custo-efetividade, sugestivas de que, apesar de seu preço mais elevado, a médio e longo prazo, sua utilização acabe por ser compensada por economia de recursos adicionalmente gastos, quando se emprega a heparina não fracionada (mais angina recorrente, e mais necessidade ulterior de intervenções coronárias). Todavia, a atual tendência de ser o antitrombínico preferido deriva de sua praticidade de administração, a $1 \mathrm{mg} / \mathrm{Kg}$, por via subcutânea, a cada 12 h, sem necessidade de controle laboratorial. Devido a maior risco de sangramento, há necessidade de controle laboratorial através da dosagem do fator Xa, mantendo-se valor entre 1,5 a 2 vezes o controle, em vigência de insuficiência renal (creatinina plasmática $>2.0 \mathrm{mg} / \mathrm{dL}$ ou clearance de creatinina $<30 \mathrm{ml} / \mathrm{min}$ ), obesidade (índice de massa corporal $>30$ ) ou idade avançada (> 75 anos). Nessas condições, a farmocodinâmica do medicamento torna-se imprevisível, portanto, na impossibilidade de se realizar verificação laboratorial dos níveis de fator Xa, deve-se utilizar heparina não fracionada.

\section{g) Inibidores da glicoproteína IIb/IIIa}

Antiplaquetários que bloqueiam a via final comum da agregação plaquetária, independente do estímulo inicial. Tiveram seu uso bem fundamentado, apenas no ambiente da intervenção coronária, percutânea, e, em menor grau, em pacientes de alto risco (especialmente diabéticos), mesmo quando tratados clinicamente. Os estudos que embasaram tais noções foram realizados, quando a ativação plaquetária era antagonizada, sistematicamente, apenas pelo AAS. Portanto, a adição de inibidores da glicoproteína IIb/IIIa ao AAS + tienopiridínico, constituindo o bloqueio plaquetário triplo, ainda carece de demonstração de que seu benefício suplante o inegavelmente mais elevado risco de sangramento. Esse conceito, aliado ao alto custo, recomenda utilização bem individualizada dos compostos, e não de rotina, principalmente, quando os pacientes não são tratados por ICP. São de uso exclusivamente EV e, em 
geral, mantidos por cerca de 12-48 h. Fora do ambiente da ICP, o tirofiban é preferível, ao passo que o abciximab parece ter se associado a melhores resultados, quando os pacientes são tratados percutaneamente.

h) Inibidores da enzima de conversão da angiotensina (IECA)

Seus efeitos positivos são bem comprovados, com disfunção ventricular conseqüente ao IAM. Embora sem demonstração direta de benefícios, em contextos isquêmicos, agudos, sem infarto, há evidências, sugerindo ação protetora, vascular, generalizada, desses inibidores, que poderiam expandir sua indicação para todas as apresentações da doença isquêmica do miocárdio, inclusive a angina instável. Constituem, também, opção adequada no controle de pacientes que se mantêm hipertensos após doses adequadas de nitratos e betabloqueadores.

\section{i) Antagonistas dos canais de cálcio}

Estão indicados nas síndromes isquêmicas, agudas, somente quando a isquemia se mostra refratária aos betabloqueadores e nitratos em doses otimizadas, ou em casos de Hipertensão Arterial, refratária; devese dar preferência aos dotados de cronotropismo negativo, como o verapamil ou o diltiazen. Devem ser utilizados com muita cautela, na presença de disfunção ventricular esquerda ou insuficiência cardíaca, mas têm indicação realçada em pacientes com vasoespasmo superimposto a placas ateroscleróticas.

j) Decisão final sobre estratégia de_avaliação de risco e eventual revascularização

Há virtual consenso de que pacientes de alto risco de complicações graves (óbito e infarto) devam, preferencialmente, ser cateterizados, e a angiografia, ainda que, muitas vezes, complementada por exames funcionais (e.g. CMP ou ECO-estresse), deve nortear, predominantemente, o caminho a ser seguido: tratamento clínico apenas, ou ICP, ou cirurgia para revascularização miocárdica. Em contraposição, os de baixo risco, conforme indicado acima, podem, em princípio, ser avaliados, inicialmente, com os exames funcionais. Para os de risco intermediário, observa-se certa tendência recente a priorizar-se o estudo anatômico e a consequiente ICP, como preferencial, na maioria dos pacientes. Mas, embora bastante persuasivos, os indícios de superioridade de tal conduta, em pacientes com risco intermediário, não são absolutamente conclusivos, e, portanto, continua perfeitamente defensável a conduta mais conservadora, nesse con- texto. As indicações para intervenção (ICP ou revascularização cirúrgica) assemelham-se às da angina estável. Pacientes triarteriais, portadores de lesão de tronco de coronária esquerda, ou de portadores de disfunção ventricular esquerda, diabéticos, devem, $a$ priori, ter indicação para revascularização cirúrgica. Como princípio geral, é fundamental que as decisões levem em conta os aspectos clínicos da apresentação da SIMI, bem como as características angiográficas e os aspectos da avaliação funcionais.

\section{4- CONDUTAS NO INFARTO AGUDO DO MIOCÁRDIO COM SUPRADESNIVELA- MENTO PERSISTENTE DE SEGMENTO ST (IAMCSST)}

Caracteriza situação clínica de extrema dramaticidade, por: a) implicar risco imediato de morte e ter história natural bastante ominosa; b) existir tratamento capaz de alterar a história natural de forma muito favorável, mas que deve ser instituído de forma absolutamente urgente, para ser eficaz.

A definição diagnóstica baseia-se em associação de: 1) dor precordial de características anginosas, intensa, em repouso, duração $\geq 20 \mathrm{~min}$, usualmente persistindo durante a consulta ao médico; 2) alteração de ECG $=$ supradesnivelamento de $\mathrm{ST}(\geq 0.1 \mathrm{mV}$, em $\geq$ duas derivações clássicas ou precordiais contíguas, ou BCRE de desenvolvimento sabidamente (quando existe ECG prévio) ou presumivelmente novo; 3) elevação de marcadores de necrose miocárdica $=\mathrm{CKMB}$ massa $>2 \mathrm{x}$ limite superior da normalidade, ou TnT (ou TnI) $>0.1 \mathrm{ng} / \mathrm{ml}$ ).

Deve-se observar que as condutas decisivas, iniciais, serão quase sempre baseadas na combinação dos dois primeiros critérios. Isso, porque a detecção de necrose miocárdica, com base nos exames sangüíneos, caracteristicamente, requer algumas horas a partir do início do quadro clínico. Em certa proporção de pacientes, inexiste o componente sintomático (e.g. alguns pacientes diabéticos), sendo o diagnóstico firmado com base nos dois últimos critérios. Quando isso ocorre, freqüentemente se desperdiça a janela temporal de oportunidade para medidas terapêuticas iniciais.

\section{Fisiopatologia}

Decorre de trombose oclusiva, em coronária subepicárdica, sobre placa aterosclerótica rota (ou com ulceração profunda). Na maioria das circunstâncias, são placas ateroscleróticas não criticamente obstruti- 
vas da luz vascular, mas com caracteríticas patológicas de instabilidade e vulnerabilidade (alto conteúdo lipídico e celular, com cápsula fina, pouca fibrose e escassa calcificação).

Também há, usualmente, condição clínica prótrombótica e pró-inflamatória, sistemicamente instalada. Isso explica a reincidência de infarto agudo em áreas miocárdicas, distintas, e, a ainda mais comum, reinfarto (na mesma área) por reoclusão da mesma lesão inicialmente acometida. Além do componente pró-trombótico, em suas vertentes de ativação plaquetária e dos fatores de coagulação, do componente próinflamatório (e.g. elevação dos níveis de proteína-Creativa), diversos mediadores, localmente e sistemicamente liberados, causam espasmo coronário de grau variável em muitos pacientes.

- Conduta imediata - a) conforme delineado antes, história clínica sumária (para caracterização do sintoma, e fatores de risco para SIMI (que tornam o diagnóstico mais provável) e para suas complicações imediatas (que afetam o prognóstico) e exame físico dirigido para verificar os sinais vitais, detectar sinais precoces de instabilização elétrica e/ou hemodinâmica, e estabelecer a ausência de contra-indicações para trombólise endovenosa, devem ser realizados imediatamente; b) obter ECG-12 (às vezes, complementado por derivações precordiais direitas e/ou posteriores); c) instalar acesso venoso para administração de fármacos e coleta de amostras sangüíneas (CK-MB massa $6 / 6 \mathrm{~h}$ até $24 \mathrm{~h}$, troponina na entrada e, pelo menos, uma vez após 4-8 h, hemograma, inclusive contagem de plaquetas, eletrólitos, creatinina, perfil lipídico); d) monitorização contínua do ritmo cardíaco; e) RX simples de tórax, equipamento móvel (essa conduta não é essencial, de imediato, porém se aplica a muitos casos para comprovar congestão pulmonar e afastar dissecção aguda de aorta)

\section{Essas medidas devem ser executadas em até $10 \mathrm{~min}$ desde a entrada do paciente na unidade de emergência.}

\section{- Condutas iniciais, de urgência}

1. Bloqueio plaquetário - administração de ácido-acetil salicílico (AAS) por absorção bucal da preparação oral infantil $-3 \mathrm{cp}=300 \mathrm{mg}$. Em pacientes com alergia ao AAS, administrar $300 \mathrm{mg}$ de clopidogrel v.o. (4cp a $75 \mathrm{mg}$ ). Administração de inbidores da GP-IIb/IIIa, fora do contexto da intervenção coronária percutânea (ICP) não é recomendável para pacientes com IAM-CESST: além do elevado custo de tais medicamentos, os estudos realizados não conseguiram definir redução de mortalidade ou da taxa de reinfarto, mas, sim, elevado risco de sangramento.

2. Oxigenoterapia - por cateter nasal, 2$4 \mathrm{~L} / \mathrm{min}$, durante as quatro a seis primeiras $h$.

3. Analgesia - com morfina (4-8mg e.v., inicialmente, doses suplementares de $2 \mathrm{mg}$ a cada $10 \mathrm{~min}$, se necessário) ou meperidina.

4. Reperfusão miocárdica - a pedra angular do tratamento a ser instituído em todos os pacientes com IAMCEST, até $12 \mathrm{~h}$ do início dos sintomas.

Trombólise mecânica, i.e., angioplastia primária (AP) - a modalidade de recanalização coronária e reperfusão miocárdica preferencial, na maioria dos contextos, desde que possa ser efetuada até 90 min decorridos do início do quadro clínico, por equipe experiente. Paradoxalmente, há, também, evidência recente de que a AP seja preferível em pacientes nos quais a reperfusão tem que ser instituída após as 4 a 6 horas iniciais do primeiro contacto médico. Finalmente, nitidamente preferencial para pacientes com choque cardiogênico (especialmente com envolvimento da face anterior do VE).

Trombólise farmacológica - na prática, a modalidade predominante de reperfusão miocárdica, por razões logísticas. Em primeiro lugar, deve-se conferir o risco de sua implementação, com base nas contra-indicações:

Contra-indicações absolutas (nunca administrar o trombolítico).

- Sangramento ativo (exceção: menstruação), ou diátese hemorrágica conhecida.

- Gravidez ou puerpério < 1 semana.

- Dissecção aguda da aorta.

- Acidente vascular, encefálico (AVE), hemorrágico ou indeterminado pregresso.

- AVE isquêmico $<$ seis meses.

- Neoplasia encefálica.

- Trauma craniano < três semanas.

- Trauma corporal ou cirurgia extensa $<$ três semanas. 
Contra-indicações relativas (decisão de trombólise individualizada para contexto)

- Hipertensão arterial sistêmica, refratária (sistólica > $180 \mathrm{mmHg}$ ).

- AVE isquêmico, transitório < seis meses.

- Doença péptica ativa.

- Endocardite infecciosa.

- Terapia anticoagulante oral.

- Insuficiência hepática.

- Punção vascular em local não compressível.

- Ressuscitação cardiorrespiratória prolongada.

Agentes trombolíticos - todo esforço para o período decorrido entre a chegada do paciente e o início da administração desses agentes < $30 \mathrm{~min}$.

1. Estreptoquinase (SK) $-1.5 \times 10^{6}$ unidades, em $100 \mathrm{ml}$ de SF $0.9 \%$ ou SG5\% em até 1 hora de infusão e.v. Esse trombolítico costuma provocar hipotensão arterial (reduzir a velocidade de infusão), reações alérgicas (controláveis na maioria dos pacientes). É contra-indicado, se administrado anteriormente, porque será neutralizado por anticorpos específicos.

2. Ativador tissular do plasminogênio recombinante (r-TPA) - 15mg em bolus inicial, seguidos de infusões e.v. a $0.75 \mathrm{mg} / \mathrm{kg}$ durante $30 \mathrm{~min}$, e depois $0.5 \mathrm{mg} / \mathrm{kg}$ durante $60 \mathrm{~min}$. Dose total não excedendo $100 \mathrm{mg}$. Recomendável para pacientes com apresentação mais tardia (> 4-6 h), uso restrito pelo alto custo. Também de eleição quando ocorre reoclusão, em pacientes tratados previamente com SK.

3. Tenecteplase (TNK-TPA) - Injeção única e.v., de acordo com o peso corporal.

$\begin{array}{cc}\text { Dose } & \text { PESO } \\ 30 \mathrm{mg} & <60 \mathrm{~kg} \\ 35 \mathrm{mg} & 60-69 \mathrm{~kg} \\ 40 \mathrm{mg} & 70-79 \mathrm{~kg} \\ 45 \mathrm{mg} & 80-89 \mathrm{~kg} \\ 50 \mathrm{mg} & >90 \mathrm{~kg}\end{array}$

Trata-se de opção mais recentemente validada, e deverá ser de escolha, quando se contemplar, sob condições propícias, a trombólise em fase pré-hospitalar.

Heparinização - essa conduta complementa, com o bloqueio plaquetário, a essência da terapêutica antitrombótica, em pacientes com IAMCSST. Sua aplicação deve ser diferenciada, de acordo com o tipo de recanalização empregado: a) é absolutamente imperativa durante o procedimento de AP, mas, rotineiramente, deve cessar após o término do procedimento; b) deve ser iniciada, simultaneamente, com a administração de r-TPA e de TNK-TPA, e continuar por 24 a $48 \mathrm{~h}$; c) para pacientes tratados com SK, fibrinolítico, não específico, quando existe baixo risco de trombose, dispensa-se a heparinização, pelo menos, durante as $12 \mathrm{~h}$ que se seguem ao início da trombólise. Essa conduta tem sido questionada, por ser lastreada em resultados de estudos clínicos mais antigos, e está, presentemente, sob escrutínio de investigações dirigidas.

Regime de heparinização - heparina não fracionada (HNF), em doses ajustadas para o peso corporal: $60 \mathrm{U} / \mathrm{kg}$ (máximo $=4000 \mathrm{U})$ como bolus inicial, seguido de infusão de 10-12 U/kg/h (máximo de 1000 $\mathrm{U} / \mathrm{h}$ ), ajustada para manter o tempo de tromboplastina parcial, ativada (TTPA) $=1.5-2.5 \times$ controle $(50-75$ seg). Monitorização do TTPA recomendável após 3, 6,12 e 24 h do início. A enoxaparina teve eficácia equivalente em estudos recentes, e, apesar da desvantagem de mais elevado custo inicial, é mais facilmente administrada: bolus inicial de $30 \mathrm{mg}$ e.v., seguidos de $1 \mathrm{mg} / \mathrm{kg}$ s.c. de 12/12 h. Em pacientes com alto risco de acidentes tromboembólicos (infartos extensos, principalmente de face anterior, trombose mural, fibrilação atrial, insuficiência cardíaca, estase venosa), a enoxaparina deve ser mantida até a alta hospitalar e, depois, substituída por anticoagulantes orais, do tipo dicumarínicos.

- Condutas complementares - sem caráter de urgência, devem, contudo, ser consideradas nas primeiras horas

a) Avaliação da função ventricular - usualmente, por ecocardiografia transtorácica, em condição basal, nas primeiras $24-48 \mathrm{~h}$.

b) Bloqueio betaadrenérgico - a ser iniciado, por via e.v., nas primeiras horas, sempre que não houver contra-indicações (bradicardia < 50/min, PR > $0.24 \mathrm{seg}, \mathrm{BAV}>1^{\circ}$ ou doença sinusal sem marca-passo cardíaco, PAS $<90 \mathrm{mmHg}$, história de doença pulmonar obstrutiva; insuficiência cardíaca descompensada; choque cardiogênico). Usar, de preferência, metoprolol, em dose de $5 \mathrm{mg}$ por injeção endovenosa durante 3-5 min, e repetir a cada $5 \mathrm{~min}$, até dose cumulativa de $15 \mathrm{mg}$. Depois, continuar com administração oral, por tempo indefinido, de 25-100 mg de succninato de metoprolol, em duas doses diárias, tituladas pela tolerância do paciente. 
c) Bloqueio da enzima de conversão da angiotensina I (ECA) ou do receptor da angiotensina II - na ausência de contra-indicações (PAS < $90 \mathrm{mmHg}$, alergia conhecida, estenose renal bilateral, insuficiência renal grave, hipovolemia) a ser implementado de rotina, mas, principalmente, se houver sinais e sintomas de disfunção ventricular esquerda, ou quando o paciente permanece hipertenso. A administração do bloqueador deve ser iniciada ainda nas primeiras $12 \mathrm{~h}$ do início do quadro de IAM, titulando-se as doses até o máximo tolerado, com alvo de: captopril $150 \mathrm{mg}$ v.o, por dia, dose inicial de $6.25 \mathrm{mg} 2-3 \mathrm{x} / \mathrm{dia}$; ramipril $10 \mathrm{mg}$ v.o., por dia, dose inicial $2.5 \mathrm{mg} 2 \mathrm{x} / \mathrm{dia}$; enalapril $40 \mathrm{mg} / \mathrm{dia}$, dose inicial $2.5 \mathrm{mg} 2 \mathrm{x} / \mathrm{dia}$; valsartan $320 \mathrm{mg} / \mathrm{dia}$, dose inicial 20 mg 2x/dia. Mesmo em pacientes com total preservação da função de VE após o IAM, um desses medicamentos (preferencialmente o ramipril, ou o perindopril), deve continuar, por tempo indefinido, como parte da prevenção secundária a longo prazo.

d) Administração de antagonista da aldosterona - no contexto do IAM, houve demonstração de que, em pacientes com alto risco de complicações (disfunção de VE e congestão pulmonar), o antagonista mais seletivo, Eplerenone, produziu discreto, mas nítido benefício, em comparação com placebo, quanto a morbimortalidade, mesmo após o bloqueio da ECA. Entretanto, esse medicamento ainda não está difundido no País, e o uso do inibidor da aldosterona, mais antigo e econômico, (espironolactona) somente foi validado para pacientes com IC de grau avançado (classes III e IV da NYHA). Assim, não se considera indicado de rotina, após o IAM, o uso desses antagonistas da aldoterona, mas será opcional sua administração, quando o eplererenone estiver disponibilizado.

e) Administração de nitratos -para pacientes sem hipotensão arterial (PAS > $100 \mathrm{mmHg}$ ) pode-se, após constatar que o supradesnivelamento de ST não é na face inferior do VE, administrar $5 \mathrm{mg}$ de dinitrato de isossorbida $\mathrm{SL}$, ou $0.4 \mathrm{mg}$ de nitroglicerina SL, como teste, para afastar a possibilidade de síndrome de Prinzmetal (espasmo coronário grave, em artéria subepicárdica proximal, importante diagnóstico diferencial de elevação - usualmente transitória - de ST). Em pacientes com supradesnivelamento de ST na face inferior, principalmente, se cursando com bradicardia elou hipotensão, nitratos não devem ser administrados, pelo risco de agravamento das condições hemodinâ- micas, afetando o desempenho de VD. Após as primeiras horas, a administração de nitratos, por via oral ou mesmo endovenosa, é justificável, para tratar a congestão pulmonar e/ou disfunção sintomática do VE, por 24-48 h. Deve ser instituído regime alternativo, de $12 \mathrm{~h}$ diárias, sem nitrato, para controlar a taquifilaxia comum nesses casos.

f) Uso de diuréticos - indicado em vigência de insuficiência cardíaca, clinicamente manifesta por congestão pulmonar e/ou sistêmica. Furosemida em doses de 20-40mg e.v. ou v.o, repetidas a cada 4-6 h

g) Administração de antagonistas dos canais de cálcio - a serem evitados na fase hiperaguda do IM. Depois, aqueles providos de tendência bradicardizante (verapamil, dialtiazen) podem ser administrados, como substitutos dos bloqueadores betaadrenérgicos, a pacientes que não toleram esses medicamentos.

h) Dieta para controle de dislipidemia, dos níveis de glicemia e uso precoce de inibidores da hidroximetilglutarilcoenzima $\mathbf{A}$ - são medidas instituídas rotineira e precocemente, como parte integrante da estratégia de prevenção secundária, a longo prazo, após o IAM. Doses únicas, diárias, de sinvastatina, pravastatina, lovastatina ou atorvastatina devem ser tituladas para serem atingidos os níveis preconizados de LDL-colesterol no sangue dos pacientes restabelecidos de IAM. Há evidência recente de benefício com esses medicamentos, mesmo com valores de LDL-C anteriormente interpretados como satisfatórios.

i) Avaliação da reserva vasodilatadora, coronária e de viabilidade miocárdica na região do IAM e em outros territórios do VE - a conduta é aplicável aos pacientes, evoluindo sem complicações, podendo ser realizada tão cedo quanto 48-72 h do início do quadro clínico. O método de eleição, nessa fase precoce, é o da cintilografia miocárdica, perfusional, com Tálio-201 ou Tc-99m-Sestamibi, em condição basal e sob ação do dipiridamol ou adenosina. Ainda em pacientes estáveis, após o $5^{\circ}$ dia de evolução, considera-se seguro realizar teste ergométrico submáximo ou farmacológico com dobutamina, monitorado por ecocardiografia. A ecocardiografia de estresse, nesse contexto, tem sensibilidade discretamente inferior e especificidade discretamente superior à cintilografia, para detectar isquemia e viabilidade miocárdicas; tem, ainda, risco de desencadear arritmias ventriculares no período após o IAM. 
Cateterismo cardíaco, estudo hemodinâmico intravascular e angiografia coronária de contraste radiológico e intervenção coronária percutânea (ICP) - além da circunstância da AP, já discutida acima, o estudo anatômico, visando a ICP ou mesmo revascularização miocárdica cirúrgica está indicado:

a) como "salvamento" para tratar pacientes nos quais a terapia trombolítica, farmacológica não produziu efeito, e persistem os sintomas e sinais de isquemia em evolução;

b) quando, após período de arrefecimento, os sinais e sintomas de isquemia reincidem, indigitando reoclusão precoce (usualmente, com reinfarto), requerendo ICP de "resgate precoce";

c) em pacientes estáveis, após trombólise farmacológica, mas com isquemia indutível, durante o estresse farmacológico ou por esforço físico;

d) em pacientes não tratados com estratégia de reperfusão (em geral, atendidos tardiamente).

Considerando-se o conjunto dos pacientes sobreviventes às primeiras horas que se sucedem a um episódio de IAMCSST, mesmo adotando-se, inicialmente, estratégia de estratificação de risco, baseada em estudos funcionais (e.g. cintilografia miocárdica de perfusão ou ecocardiografia de estresse), é previsivel que, em $\geq 80 \%$ das instâncias o estudo anatômico da circulação coronária com angiografia de contraste termine por ser necessário. Entretanto, duas observações, no contexto, são relevantes: a) em muitos pacientes o grau de estenose assim verificado, na artéria relacionada ao evento isquêmico é potenciado por trombose potencialmente reversível ao cabo de poucos dias; b) em diversas circunstâncias de lesões moderadamente obstrutivas (faixa de 40-60\% de redução do diâmetro luminal), o exame cintilográfico ou ecocardiográfico será requerido para se avaliar o significado funcional da obstrução limítrofe.

Durante ICP, as endoprotóteses coronárias (stents) são, atualmente, implantadas em > 90\% dos pacientes, quase sempre visando à lesão na artéria relacionada ao evento. Isso, a despeito de alarma inicialmente verificado, de resultados de mortalidade mais elevada com esses dispositivos comparativamente à dilatação simples com balão: essa atual predileção pelo emprego dos stents apóia-se em resultados melhores, recentemente, muito provavelmente devidos à experiência dos intervencionistas, ao aperfeiçoamento do material das endopróteses (mesmo sem uso generalizado das endopróteses eluidoras de substâncias antiproliferativas), e à melhor terapêutica adjunta. Em tal contexto, o bloqueio duplo da ativação plaquetária com AAS + tienopiridínico (ticlopidina $250 \mathrm{mg} 2$ x/dia ou clopidogrel $75 \mathrm{mg} /$ dia v.o.) é imperativo para todos os pacientes. Embora ainda não haja demonstração específica de que, em pacientes cuja ativação plaquetária esteja bloqueada na via da ciclo-oxigenase (pelo AAS) e na via do ADP (pelo tienopiridínico), o uso de bloqueadores da via final de agregação plaquetária confirma benefício suplementar, sem incorrer em risco intolerável de sangramento, e de que os custos dessa conduta sejam aceitáveis, o abciximab (preferível, $0.25 \mathrm{mg} / \mathrm{kg}$, inicial, seguidos de infusão por $12 \mathrm{~h}$, de $0.125 \mu \mathrm{g} / \mathrm{kg} / \mathrm{min}$, máximo de $10 \mu / \mathrm{min})$ ou o tirofiban $(10 \mu \mathrm{g} / \mathrm{kg} / \mathrm{min}$, inicial, seguidos de $0.15 \mu \mathrm{g} / \mathrm{kg} / \mathrm{min}$, durante $18-24 \mathrm{~h}$ ) podem ser indicados para alguns pacientes que estão sendo tratados com ICP. Quando esses inibidores da GPIIb/IIIa são empregados, torna-se imperativo reduzir as doses de heparina utilizadas, bem como é mandatório o ajuste para pacientes mais idosos ou com função renal deprimida, quando se usa heparina fracionada.

MARIN-NETO JA; MACIEL BC; PAZIN FILHO A \& CASTRO RBP. Acute Coronary Syndromes in the Emergency Department. Medicina, Ribeirão Preto, 36: 187-199, apr./dec.2003.

ABSTRACT - Acute Coronary Syndromes (ACS) are very frequent in the emergency department and the lack of proper diagnosis or inadequate treatment imply great morbidity and mortality. The ACS treatment is dependent on the risk, estimated by medical history and physical examination, eletrocardiographic parameters and serologic necrosis markers. The therapeutic principles for ACS with ST elevation (acute myocardial infarction) and of the ACS without ST elevation (unstable angina and acute myocardial infarction without ST elevation) are reviewed.

UNITERMS - Myocardial Ischemia. Myocardial Infarction. Angina, Unstable. 


\section{BIBLIOGRAFIA RECOMENDADA}

1 - AMERICAN COLLEGE OF EMERGENCY PHYSICIANS. Clinical Policy for the initial approach to adults presenting with a chief complaint of chest pain, with no history of trauma. Ann Emerg Med 25: 274-299, 1995.

2 - JESSE RL \& KONTOS MC. Evaluation of chest pain in the emergency department. Curr Prob Cardiol 22: 149-236, 1997.

3 - MEHTA RH. Missed diagnoses of acute coronary syndromes in the emergency room continuing challenges. $\mathbf{N}$ Engl J Med 342: 1207-1210, 2000.

4 - EWY GA \& ORNATO JP. 31st Bethesda Conference. Emergency Cardiac Care. Task force 1: Cadiac arrest. J Am Coll Cardiol 35: 825-880, 2000.

5 - LEE TH \& GOLDMAN L. Primary care: Evaluation of the patient with acute chest pain. N Engl J Med 342:11871195, 2000.

6 - ANTMAN EM \& BRAUNWALD E. Acute myocardial infarction. In: BRAUNWALD E, ed. Textbook of cardiovascular medicine. Saunders, Philadelphia, p.1114-1231, 2001.

7 - CANNON CP \& BRAUNWALD E. Unstable angina. In: BRAUNWALD E, ed. Textbook of cardiovascular medicine. Saunders, Philadelphia, p. 1232-1271, 2001.

8 - NICOLAU JC \& MARIN-NETO JA, ed. Síndromes Isquêmicas Miocárdicas Instáveis. Ed. Atheneu, São Paulo, 2001.

9 - BASSAN R; PIMENTA L; LEÃES PE \& TIMERMAN A. SOCIEDADE BRASILEIRA DE CARDIOLOGIA. I Diretriz de dor torácica na sala de emergência. Arq Bras Cardiol 79: 121, 2002. Supl. II
10 - BRAUNWALD E; ANTMAN EM; BEASLEY JW; CALIFF RM; CHEITLIN MD; HOCHMAN JS; JONES RH; KEREIAKES D; KUPERSMITH J; LEVIN TN; PEPINE CJ.; SCHAEFFER JW; SMITHEE $3^{\text {rd }}$; STEWARD DE; THEROUXP;ALPERT JS; EAGLE KA; FAXON DP; FUSTER V; GARDNER TJ; GREGORATOS G; RUSSELL RO \& SMITH SC JR. ACC/AHA guidelines for the management of patients with unstable angina and non-STsegment elevation myocardial infarction. J Am Coll Cardiol 36: 970-1062, 2002.

11 - ERHARDT L; HERLITZ J; BOSSAERT L; HALINEN M; KELTAI M; KOSTER R; MARCASSA C; QUINN T \& van WEERT H. Task force on the management of chest pain. Eur Heart $\mathbf{J}$ 23: 1153-1176, 2002.

12 - NICOLAU JC; CESAR LAM; TIMERMAN A; PIEGAS LS \& MARIN-NETO JA. Diretrizes da Sociedade Brasileira de Cardiologia sobre angina instável e infarto agudo do miocárdio sem supradesnível do segmento S-T: Parte I Estratificação de risco e condutas nas primeiras $12 \mathrm{~h}$ após a chegada do paciente ao hospital. Arq Bras Cardiol 77: 3-23, 2001. Supl. II.

13 - FEITOSA GS; NICOLAU JC; PIEGAS, LS; TIMMERMAN A \& MARIN NETO JA. Diretrizes da Sociedade Brasileira de Cardiologia para IAM com supra-desnivelamento de ST. Arq Bras Cardiol 74: 1-46, 2000. Supl. II.

14 - MACIEL BC \& MARIN-NETO JA, eds. Manual de condutas clínicas cardiológicas. Ed. Segmento Pharma, São Paulo, 2004. 